\title{
Transesterification in epoxy-thiol exchangeable liquid crystalline elastomers
}

\author{
Alexandra Gablier, Mohand O. Saed, Eugene M. Terentjev*
}

Cavendish Laboratory, University of Cambridge, Cambridge CB3 OHE, U.K.

KEYWORDS: liquid crystal elastomers, thiol-epoxy, transesterification, vitrimer, thermal actuation

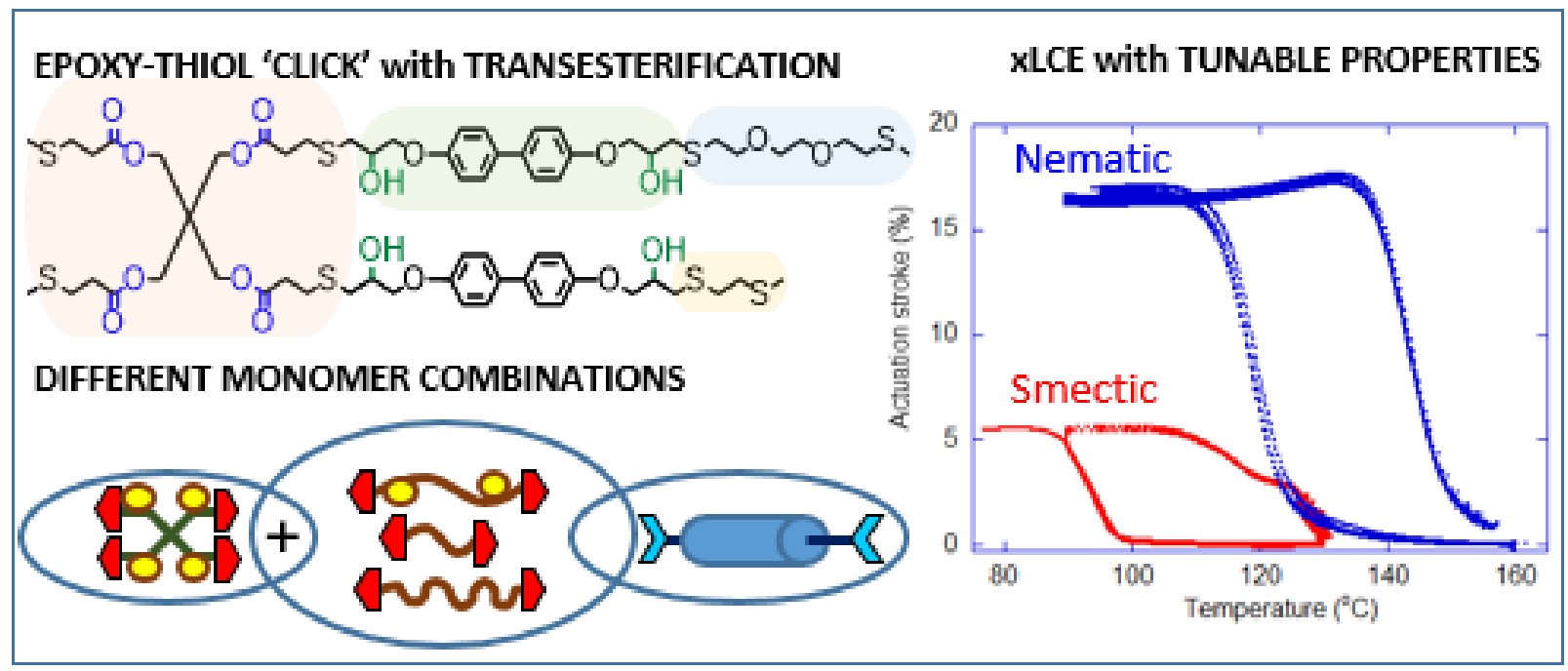

\footnotetext{
ABSTRACT: The incorporation of vitrimer bond-exchange chemistry into liquid crystalline elastomer networks produces 'exchangeable liquid crystal elastomers' (xLCE). These materials offer a facile method of material re-shaping and alignment postpolymerisation via the application of a mechanical stress above the temperature of activation of bond exchange. We use diepoxy mesogenic monomers with thiol-terminated spacers and crosslinker to investigate a range of resulting xLCE. The "click" chemistry of thiols results in good control over the network topology, and low glass transition in this family of materials. By combining different spacers, we were able to obtain smectic and nematic phases, and adjust the liquid crystal to isotropic phase transition between 42 and $140{ }^{\circ} \mathrm{C}$, while the elastic-plastic transition temperature was maintained close to $200{ }^{\circ} \mathrm{C}$. The broad gap between these temperatures ensures that thermally actuating uniformly aligned elastomers are stable and show no residual plastic creep, making epoxy-thiol xLCE promising for a range of engineering applications.
} 


\section{INTRODUCTION}

Liquid crystalline elastomers (LCE) have attracted a lot of attention since their conceptualisation ${ }^{1,2}$ for their ability to reversibly change their equilibrium shape, with a considerable amplitude, using stimuli that affect the underlying orientational ordering, such as temperature, ${ }^{3}$ light ${ }^{4}$ or other external fields, ${ }^{5}$ resulting in highly mechanically capable materials for actuation. ${ }^{6}$ This ability, which makes them unique in the field of "smart polymers", has made LCE highly promising components for applications ranging from soft robotics ${ }^{7}$ to sensors ${ }^{8}$, surface coatings ${ }^{9,10}$ and tissue engineering. ${ }^{11,12}$

The mechanical performance of LCE, and their key property of reversible large-amplitude actuation, are dependent on the constituting chemistry as well as the alignment of mesogenic components within the network in its equilibrium state at operating temperatures. ${ }^{13}$ Multiple strategies have been used to generate stable permanent alignment within LCE networks, ranging from surface alignment ${ }^{14}$ to photoalignment ${ }^{15}$, although historically the first and the most widely used is the mechanical alignment of samples during the final steps of polymerisation ${ }^{3,16}$ (also known as two-step crosslinking). Building arbitrary 3D structures that are aligned in specific complex patterns to actuate in a predetermined manner on a macroscopic scale is, however, not easily achieved by conventional alignment techniques. The most promising methods to obtain such aligned macroscopic structures with complex shapes have proven to be the patterned surface alignment ${ }^{17}$ and 3D printing ${ }^{18-20}$, although both methods have strong limitations on the practical thickness of the LCE film. Exchangeable liquid crystalline elastomers (XLCE) ${ }^{21}$ have introduced an entirely new method of preparing complex shapes of xLCE and 'programming' their alignment (and therefore, the actuation patterns). xLCE are highly promising and versatile materials with sustainable devices and realistic industrial applications in mind.

xLCE were rendered possible through the use of the generic concept of vitrimers ${ }^{22}$ - covalently bonded polymer networks, in which associative bond-exchange reactions (BER) can be activated above a certain temperature. Above such a 'vitrification temperature', the network anisotropy needed for the alignment of XLCE becomes possible through the plastic flow due to reshuffling of network bonds in the material under stress. This new anisotropic state is then locked into the network once the temperature decreases and the exchange reactions become kinetically unfavourable, making the new network structure stable at operating temperatures. Hence, the dynamic covalent bonds incorporated within the network of xLCE offer substantial benefits in material processing by allowing the alignment to be programmed post-polymerisation, in samples of any shape or dimension. This process is additionally reversible: re-heating the samples above the vitrimer transition temperature again enables a complete reprogramming of the alignment, as well as a reshaping of the sample. This reversibility of both alignment and shape is a highly pertinent point considering the modern focus on recycling and sustainability: vitrimer chemistry yields materials that are truly renewable. ${ }^{23}$ Sample reprogramming, welding, and remoulding ${ }^{24}$ is a feat that was not attainable with 
traditional techniques of sample preparation and alignment by permanent crosslinking, including the current 3D printing. It additionally removes the constraint of having to predetermine the director pattern prior to material polymerisation, or relying on moulds to obtain complex shapes. ${ }^{25}$

The first example of xLCE was introduced in 2014 in the form of a transesterification-based elastomer obtained via an epoxyacid polymerisation ${ }^{21}$, analogous to the original isotropic vitrimer work. ${ }^{22}$ Other polymerisation and exchange chemistries have been later explored to produce LC vitrimers (xLCE), including boronic-ester exchange ${ }^{26}$ and siloxane exchange. ${ }^{27,28}$ These chemistries, for the most part, are still unexplored. Alternative systems with the scope of post-polymerisation alignment in LCE were also conceived using either disulfide exchange reaction ${ }^{29}$ or allyl sulfide RAFT20,30,31 as the exchange reaction. However, these materials do not offer the same renewability as a genuine xLCE. For instance, the dissociative nature of disulfide exchange results in a gradual deterioration of material properties due to the fluctuation of the total amount of bonds within the network when bond exchange is activated. Similarly, the radical nature of the allyl disulfide reaction entails a limit to how many times the material can be reprocessed due to a quenching of the radicals over time.

The transesterification-based xLCE system is currently the most used in the field, $21,24,25,32-38$ having demonstrated compatibility with a range of stimuli, and produced a range of applications relying on welding and reprogramming. No creep at operating temperature was reported in the original xLCE, ${ }^{21}$ however, this issue is inherent in standard vitrimers. ${ }^{26,39,40}$. Indeed, the residual bond exchange cannot be ignored even below the arbitrary-defined vitrification temperature. It is possible that the smectic liquid crystalline order in the original xLCE materials, ${ }^{21}$ made the system much stiffer and resistant to shape change. ${ }^{41-}$ 43

However, and despite the demonstrated appeal, the epoxy-acid chemistry used for this original xLCE system results in a high glass transition $\left(\mathrm{T}_{\mathrm{g}} \sim 55^{\circ} \mathrm{C}\right)$. As a result, this material is not well suited for applications at ambient temperatures. Additionally, the epoxy-acid polymerisation itself requires high temperature and extended times, and also results in the inherent branching of network topology, which reduces control over the final material properties.

Thiol chemistry has proven to be very attractive for polymer synthesis ${ }^{16,44-48}$ due to its 'pseudo-click' character, resulting in an ease of implementation, high conversion rates, and mild reaction conditions. ${ }^{49}$ Additionally, resorting to a thiol-based chemistry over an oxygen-based chemistry (e.g. in the epoxy-acid reaction) is expected to lower the glass transition of the final polymer networks. ${ }^{50,51}$ 


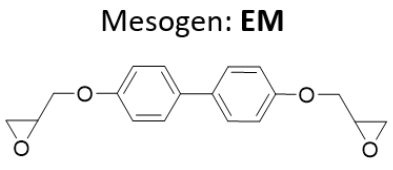

Crosslinker: PETMP

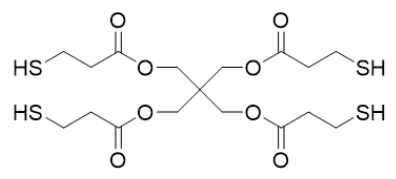

Spacer:

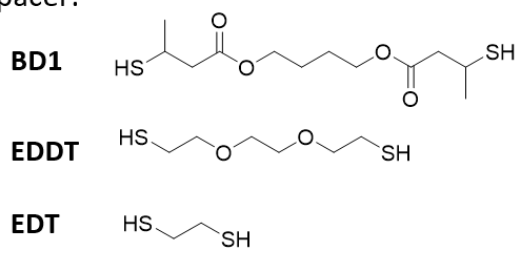

Catalyst: TBD

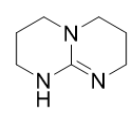

Scheme 1. Monomers and catalyst used for the xLCE network formation. An epoxy mesogen EM was reacted in equimolar quantities of functions with thiol monomers, 15\% of crosslinker PETMP and $85 \%$ of spacer, in the presence of TBD as catalyst ( $2.5 \mathrm{~mol} \%$ of epoxy function quantity).

Within thiol chemistry, the epoxy-thiol reaction has shown promise in recent works in the field of polymer and vitrimer syn-

thesis. ${ }^{49,52-56}$ It is compatible with the epoxy-terminated mesogenic monomer (EM in Scheme 1) used in previous xLCE studies involving transesterification. EM presents the advantage of being stable during the bond exchange, as it does not contain any ester groups within its structure; this is a prerequisite to ensure renewability for transesterification-based xLCE. In contrast, the presence of ester groups within the mesogen would lead to a progressive destruction of the mesogen through exchange reactions. ${ }^{36}$

Hence the epoxy-thiol chemistry appeared as a promising approach to material synthesis of xLCE as well. In contrast to the more commonly used epoxy-acid polymerisation, the crosslinking in the epoxy-thiol system is only produced during polymerisation by the fixed amount of four-functional thiol monomer, as there exists no competing side reaction. The network obtained post-polymerisation is hence expected to present an overall more controlled and homogeneous structure. However, once the network is brought above the BER activation temperature, new three-functional crosslinks form through the chain branching from the hydroxyl-ester transesterification, until a new equilibrium network topology is attained after a sufficient time for the reaction (representing a mixture of 4-functional thiol crosslinkers, 3-functional chain branching points, and the corresponding dangling ends acting as the network plasticiser). ${ }^{57,58}$

Using this polymerisation chemistry, the $-\mathrm{OH}$ groups required for the transesterification BER are generated in situ through the epoxy ring opening reaction during the polymerisation (Scheme 2). The ester groups on the other hand are incorporated into the system only through the structure of selected thiol monomers (spacers and crosslinker, Scheme 1). This enables good control over the amount of functional groups participating in the bond exchange. A minimal amount of ester was introduced 

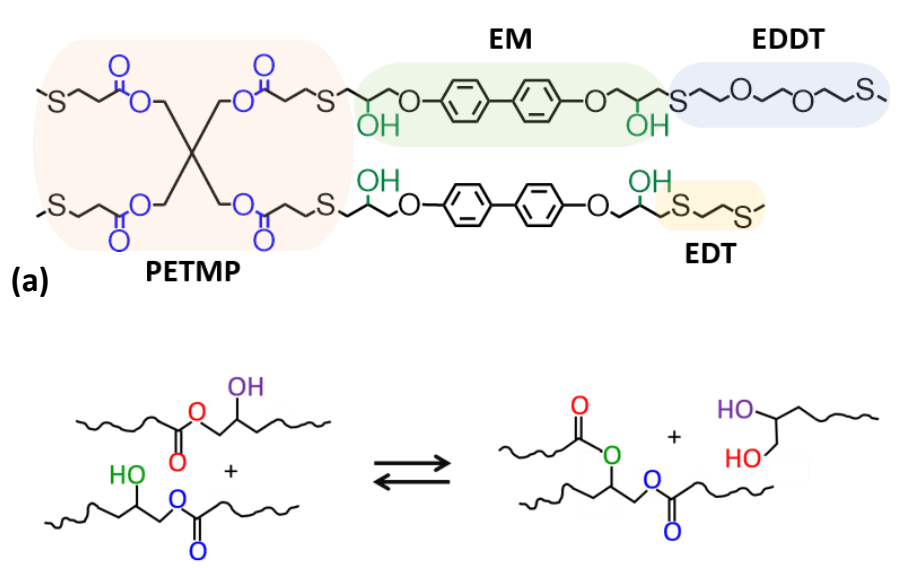

(b)

Scheme 2. (a) Network structure for a network composed of a mix of spacers (EDDT and EDT). The reactive groups for the BER are marked in colour; (b) Transesterificaction in the polymer network can result in the appearance of a new crosslink and a dangling chain.

in all networks through the structure of the thiol-based crosslinker (PETMP). Three different thiol-based spacers were explored in this work: an ester-containing spacer with a tendency to hinder mesogen alignment (BD1), and two ester-free spacers: one long (EDDT) and one short (EDT), see Scheme 1. In this way, by changing the composition, we design a family of related xLCE materials with a low glass transition $\mathrm{T}_{\mathrm{g}}$, and a controlled network structure, while preserving the same bond exchange process that was the source of the renewability in the original XLCE. This in turn leads to new xLCE which display a diverse range of equilibrium characteristics and actuation properties.

\section{EXPERIMENTAL SECTION}

Materials. All reagents were sourced from commercial suppliers, and used as received unless specified otherwise. The catalyst 1,5,7-triazabicyclo[4.4.0]dec-5-ene (TBD), the crosslinker pentaerythritol tetrakis (3-mercaptopropionate) (PETMP), and the thiol spacers 1,2-Ethanedithiol (EDT) and 2,2'-(Ethylenedioxy) diethanethiol (EDDT) were purchased from SigmaAldrich. 1,4-Bis (3-mercaptobutyryloxy)butane (BD1) was provided by Showa Denko. The epoxy mesogen (EM) was synthesised following literature procedures. ${ }^{59,60} \mathrm{TBD}$ was stored in a desiccator, as it is known to be hygroscopic and its reactivity is adversely affected by humidity. For the same reason, all polymerisation reactions were performed in dry solvent. Anhydrous dimethylformamide (DMF) was purchased from Sigma-Aldrich and used as received. Purity of reagents was accounted for when calculating experimental masses for all monomers for polymerisations.

Preparation of the polymer networks. The epoxy monomer (EM, 100\% of epoxy functional groups, $0.5 \mathrm{~g}, 3.35 \mathrm{mmol}$ ), crosslinker (PETMP, 15\% of thiol functions, $0.065 \mathrm{~g}, 0.503 \mathrm{mmol}$ ), and EDDT ( $85 \%$ of thiol functions, $0.273 \mathrm{~g}, 2.85 \mathrm{mmol}$ ) 
were dissolved in anhydrous DMF $(1 \mathrm{~mL})$ at $80^{\circ} \mathrm{C}$. Under vigorous stirring, the catalyst solution (TBD, $2.5 \mathrm{~mol} \%$ to the epoxy groups, $11 \mathrm{mg}, 0.085 \mathrm{mmol}$, dissolved in $100 \mu \mathrm{L}$ of anhydrous DMF) was added. The mixture is poured into a PTFE mould and left to polymerise at $80^{\circ} \mathrm{C}$ for multiple hours. After the polymerisation was completed, samples were transferred to a vacuum oven at $80^{\circ} \mathrm{C}$ for $24 \mathrm{~h}$ to ensure complete solvent removal. The final material appears as a white opaque elastomeric network, reflecting its natural polydomain LCE texture. Since EDT is a low-molecular weight compound, volatile stench chemical, we carried the reaction in the vacuum-sealed bags using the Toyuugo food vacuum sealer.

Constant-force ("iso-stress") experiments. In the literature, these tests are often referred to as "dilatometry", which is a wrong term since the volume of the polymer network stays strictly constant. We used the TA Discovery DMA 850 instrument in tensile mode. The samples were equilibrated at a starting temperature of $30^{\circ} \mathrm{C}$, after which the stress of $50 \mathrm{kPa}$ was applied; the resulting extensional strain was a reflection of the tensile modulus of each network. The extensional strain of a sample under constant tensile stress was then monitored as they were subject to a ramp in temperature of $2{ }^{\circ} \mathrm{C} / \mathrm{min}$, as the material started to flow at a certain temperature, increasing its length at constant engineering stress.

Monodomain programming and actuation measurements. Monodomain programming was performed on a TA DMA Q800 in the creep test mode. The sample was held under a constant uniaxial stress of $100 \mathrm{kPa}$ at $170{ }^{\circ} \mathrm{C}$ until a $100 \%$ strain was achieved via creep, after which it was cooled to room temperature. In another similar process, with a sample that had a higher vitrification temperature, we held the stress of $200 \mathrm{kPa}$ at $200{ }^{\circ} \mathrm{C}$, in which case the prescribed $100 \%$ strain was reached faster. To measure its actuation, the sample was cycled multiple times between $90^{\circ} \mathrm{C}$ and $130{ }^{\circ} \mathrm{C}$ at $2-3{ }^{\circ} \mathrm{C} / \mathrm{min}$ under a constant low stress of $50 \mathrm{kPa}$ to preserve the taut configuration.

Wide angle x-ray scattering (WAXS). WAXS was performed on a Phillips PW1830 generator equipped with Gemstar imageintensified CCD area detector. The X-ray images were inverted from black to white for better visibility.

Other characterisation. Differential scanning calorimetry (DSC) analysis was done on a Perkin Elmer DSC 4000 instrument. A temperature ramp of $10^{\circ} \mathrm{C} / \mathrm{min}$ was used. Samples were subjected to a heating-cooling-heating cycle from -50 to $200{ }^{\circ} \mathrm{C}$; data was extracted from the second heating run. Infrared spectrometry (FTIR) data were recorded between $400-4000 \mathrm{~cm}^{-1}$ on a Thermo Scientific Nicolet iS10 spectrometer using KBr Real Crystal IR sample cards and cover slips. For monitoring the polymerisation, the disappearance of the thiol peak was used as tracker of the advancement of the reaction rather than the epoxy peak, as it was reported to not be appropriate for such a purpose. ${ }^{61}$ 


\section{RESULTS AND DISCUSSION}

Epoxy-thiol "click" chemistry. An epoxy-thiol reaction was selected for the network synthesis. Polymerisation typically occurred at $80^{\circ} \mathrm{C}$ in DMF over the course of a couple of hours, the time depending on the spacer used. Reaction progress was monitored by FTIR. Solvent was used to slow down the reaction speed and lower the crystallisation temperature of the mesogenic EM molecule to $75^{\circ} \mathrm{C}$. At such a temperature, no epoxy homo-polymerisation is expected to occur despite the presence of a base catalyst; 50,54 this was confirmed by FTIR results (Fig. 1), which show a total consumption of the thiol groups which were incorporated in equimolar quantities with the epoxy groups. Note that polymerisation with the same chemistry with a closely related but non-mesogenic epoxy monomer, was taking place at ambient temperature in just 30 min; 55 the more demanding conditions in the present case are due to the restrictions of p-p stacking of the mesogenic EM. Nevertheless, these reaction conditions are overall much milder than the epoxy-acid polymerisation conditions for the original xLCE, which occurs at $180{ }^{\circ} \mathrm{C}$ for $4 \mathrm{~h}$ in a hot press. ${ }^{21}$

Using our procedure, a range of materials were synthesised using different spacer compositions. The catalyst concentration and crosslinker amount were kept constant across all samples to allow comparison. The samples were identified by their spacer content.

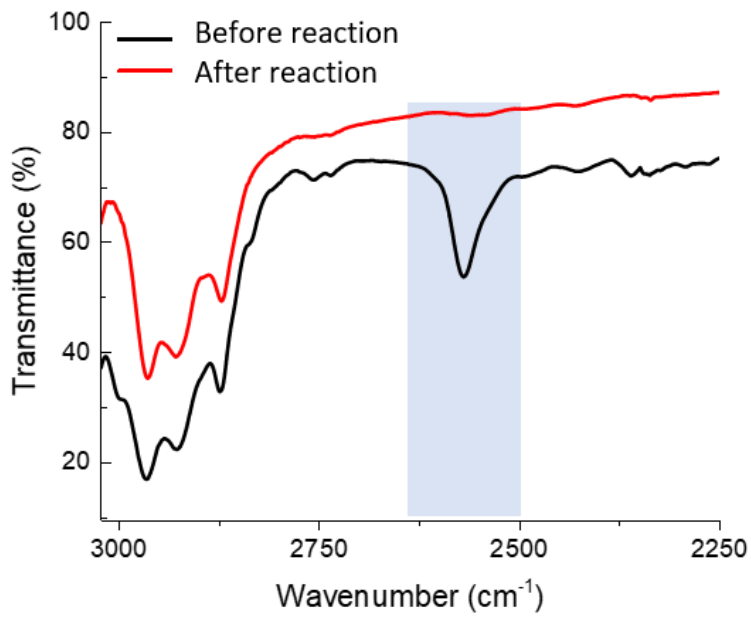

Figure 1. Reaction completion for the epoxy-thiol polymerisation was monitored via FTIR by monitoring the thiol peak at $2565 \mathrm{~cm}^{-1}$. A complete disappearance is observed, the residual hump visible on the red curve corresponding to an underlying signal originating from the internal structure of the mesogen. 
Phases and transition temperatures. xLCEs are characterised by three temperatures: their glass transition temperature $\mathrm{T}_{\mathrm{g}}$ their isotropic transition temperature $\mathrm{T}_{\mathrm{i}}$ at which the material will actuate, and the temperature of their plastic flow activation $\mathrm{T}_{\mathrm{f}}$ defined as the point at which the network begins to flow rapidly in a manner reminiscent of a liquid in the time-scale of the experiment due to the rapid ongoing BER. The glass transition and isotropic transition temperatures are measured through DSC (Fig. 2), while the $\mathrm{T}_{\mathrm{f}}$ is measured through iso-stress experiments.

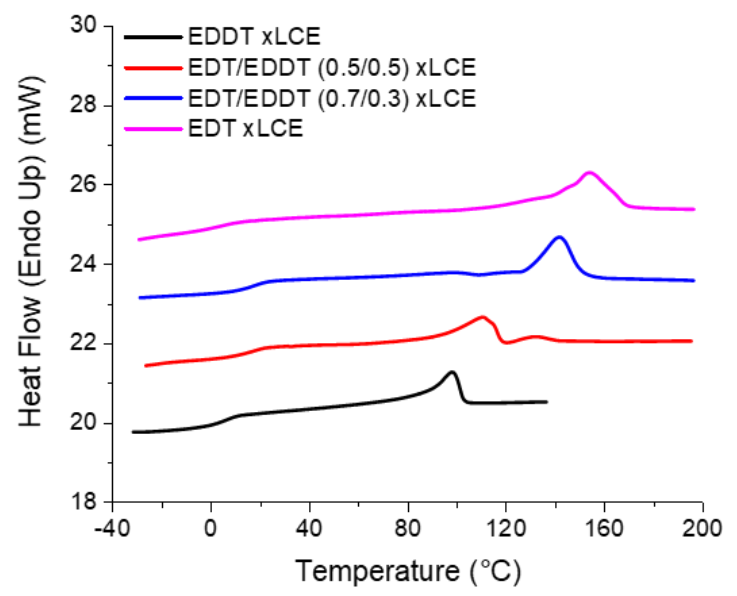

Figure 2. DSC scans of different materials identified by their constituting spacer. Two transitions are apparent from left to right: the glass transition, and the LC-to-isotropic transition.

All materials, irrespective of the spacer used, displayed a glass transition below room temperature (Table 1), making these xLCEs proper elastomers. The $\mathrm{T}_{\mathrm{g}}$ of all 'pure' systems, containing only the EDT, EDDT, or BD1 spacers, was below $6{ }^{\circ} \mathrm{C}$, while the glass transition for materials containing the random mix of spacer EDT/EDDT was about $10{ }^{\circ} \mathrm{C}$ higher. These temperatures are more than $40{ }^{\circ} \mathrm{C}$ lower than the $\mathrm{T}_{\mathrm{g}}$ reported previously for transesterification-based xLCEs. ${ }^{21}$

\begin{tabular}{l|ccc}
\multicolumn{1}{c}{$\mathrm{T}_{\mathrm{g}}$} & $\mathrm{T}_{\mathrm{i}}$ & $\mathrm{T}_{\mathrm{f}}$ \\
\hline BD1 & $5.5^{\circ} \mathrm{C}$ & $42{ }^{\circ} \mathrm{C}$ & $215^{\circ} \mathrm{C}$ \\
& & & \\
EDDT & $4.3^{\circ} \mathrm{C}$ & $90^{\circ} \mathrm{C}$ & $240{ }^{\circ} \mathrm{C}$ \\
EDT/EDDT $(0.5 / 0.5)$ & $13.8{ }^{\circ} \mathrm{C}$ & $106{ }^{\circ} \mathrm{C}$ & $265^{\circ} \mathrm{C}$ \\
EDT/EDDT $(0.7 / 0.3)$ & $13.4{ }^{\circ} \mathrm{C}$ & $127^{\circ} \mathrm{C}$ & $265^{\circ} \mathrm{C}$ \\
EDT & $4.2^{\circ} \mathrm{C}$ & $140{ }^{\circ} \mathrm{C}$ & $260{ }^{\circ} \mathrm{C}$
\end{tabular}

Table 1. Glass transition temperature $\left(T_{g}\right)$, isotropic transition temperature $\left(T_{i}\right)$, and flow temperature $\left(T_{f}\right)$ for the xLCE materials. The values of $T_{f}$ are with higher uncertainty of $\pm 5^{\circ} \mathrm{C}$. 
The nature of the spacer used showed the strongest impact on the transition to the isotropic phase, $\mathrm{T}_{\mathrm{i}}$. DSC experiments (Table 1) show that the $T_{i}$ could be made to cover a range from near room temperature to $140{ }^{\circ} \mathrm{C}$, depending on the spacer used in the network structure. A material based on the bulky BD1 displayed an isotropic transition temperature of just $42{ }^{\circ} \mathrm{C}$, while an EDDT-based material had a $\mathrm{T}_{\mathrm{i}}$ of $90^{\circ} \mathrm{C}$. This could be further increased to $140{ }^{\circ} \mathrm{C}$ when the short EDT was the spacer between the mesogens within the network. Transitions in between these temperatures are obtained through a combination of these spacers in different proportions (Fig. 2). For example, an equimolar mix of EDT and EDDT resulted in a transition at $106{ }^{\circ} \mathrm{C}$, while an increase of the proportion of EDT to $70 \%$ shifted the transition at $127^{\circ} \mathrm{C}$. Hence, depending on the choice of spacer system, the actuation temperature could be made to cover a wide range of temperatures. This illustrates the ability to modulate the $\mathrm{T}_{\mathrm{i}}$ of this family of xLCE through small changes in the chemistry of spacers within the network, with a low impact on the glass transition.

We found that the liquid-crystalline phase in our xLCE is mostly smectic, as was the case in the original studies of Carfagna et al., ${ }^{62}$ as well as Kessler et al. ${ }^{59}$ and Ji et al. ${ }^{21}$ using the same di-epoxy biphenyl mesogenic monomer. Varying the spacer composition within this system can disrupt the smectic ordering of mesogens and change the nature of the liquid crystalline phase. Mixing spacers of different lengths is a known technique to decrease the lamellar propensity of polymer chains, thus promoting nematic ordering instead. With this aim in mind, the liquid crystalline phase of the xLCE materials with different proportions of the short (EDT) and the long (EDDT) spacer were studied through X-Ray diffraction in monodomain uniaxially aligned samples. With the increase in content of the short EDT spacer, we see a marked change in the nature of the ordered phase (Fig. 3). The sample with 100\% EDDT yields a complex diffraction pattern, demonstrating smectic layer reflections at small angles, alongside the orientational (nematic) reflections at wide angles. A similar smectic structure is found in the $100 \%$ EDT sample. 
(a)

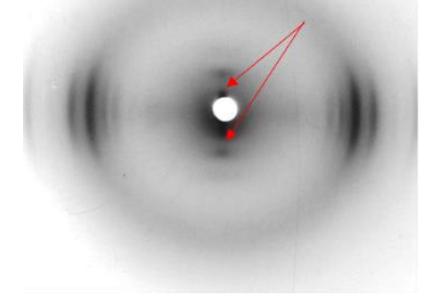

(b)

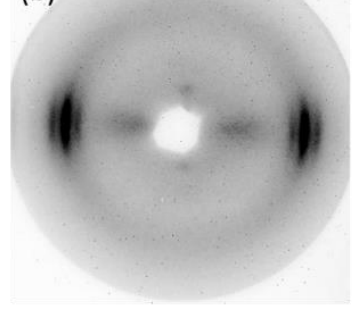

(c)

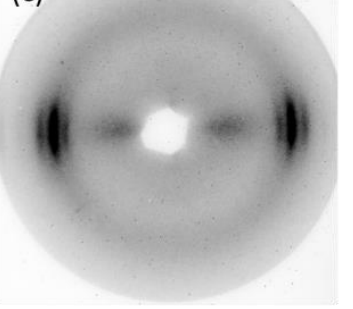

(d)

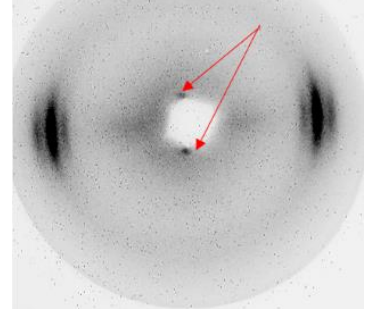

Figure 3. XRD maps of four XLCE samples, all aligned with the director vertical in the picture: (a) EDDT (100\%); (b) EDDT/EDT (0.5/0.5); (c) EDDT/EDT (0.3/0.7); (d) EDT (100\%). Patterns of pure materials (a) and (d) reveal a clear smectic phase, with layer reflections pointed by arrows. In contrast, materials with mixed spacers show less pronounced layering, with pattern (c) showing a purely nematic ordering.

As expected in the smectic phase, the orientational order parameter is high in both these materials: $Q \sim 0.86$ is obtained from the distribution of azimuthal intensity in wide-angle reflection in Fig. 3. When a mixed composition of flexible spacers is introduced, we see a much reduced smectic tendency. In particular, the 70\% EDT sample has a clear nematic phase, with the trace of smectic layers disappearing from the X-ray scattering map. In this case the orientational order parameter was much lower: $\mathrm{Q} \sim 0.64$. We are unclear about the reasons for some additional structure of the wide-angle nematic peaks, most likely originating from alignment of the second axis of flat biaxial EM mesogens. The suppression of smectic layering of EM is an unusual, previously un-reported feature of our materials..$^{21,59,62}$

Elastic-plastic transition. The plastic flow of the xLCE, produced by transesterification bond exchange activated by temperature and applied stress, was investigated through iso-stress experiments (Fig.4). Similarly to the way the glass transition measured in a DSC is dependent on the heating rate applied during an experiment, the $\mathrm{T}_{\mathrm{f}}$ values measured through iso-stress testing are also dependent on the heating rate, and the value of constant stress applied to the sample. ${ }^{38} \mathrm{As}$ such, $\mathrm{T}_{\mathrm{f}}$ represents

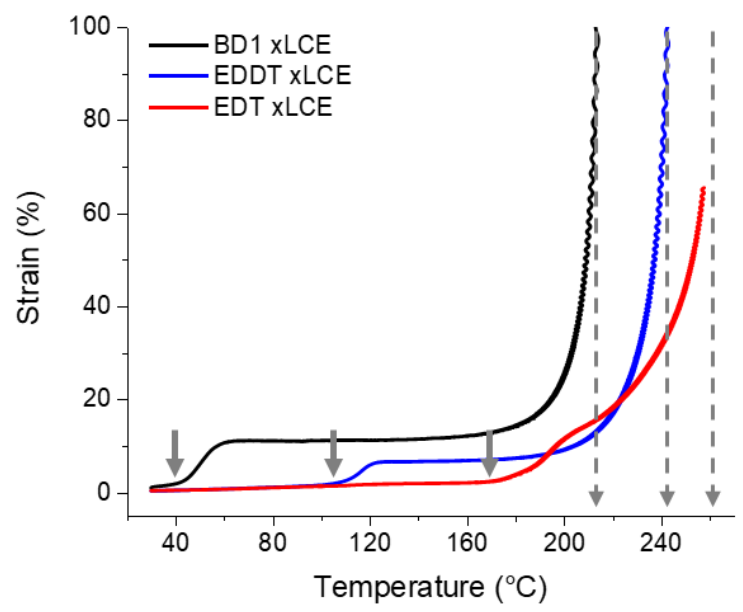

Figure 4. Iso-stress experiments for BD1, EDDT and EDT samples. The $T_{i}$ is apparent through a change in material properties from the smectic to the isotropic state as indicated by the short grey arrows; the $T_{f}$ is obtained as the temperature at which the material flows rapidly (indicated by the dashed arrows). 
only an indicative measure of the plastic flow phenomenon rather than a definitive point of 'vitrification. A low stress value $(50 \mathrm{kPa})$ and heating $\operatorname{ramp}\left(2^{\circ} \mathrm{C} / \mathrm{min}\right)$ were used in this test.

In our earlier work on isotropic epoxy-thiol vitrimers, we have characterised the kinetics of the hydroxyl-ester BER, the results of which are relevant to the present study. ${ }^{55}$ Using a very similar (but non-mesogenic) di-epoxy monomer and exactly the same thiol spacers, crosslinker, and catalyst, we found that for the EDDT spacer (where the only source of esters is the PETMP crosslinker, while every EM monomer provides an -OH group at each end), the activation energy of BER is $134 \mathrm{~kJ} / \mathrm{mol}$, while for the BD1 (where in addition, two more ester groups are available on each spacer) the activation energy is $92 \mathrm{~kJ} / \mathrm{mol}$. BD1 network also was shown to have the lower rubber modulus in the isotropic phase; both factors made the elastic-plastic transition in the BD1 vitrimer occur at a lower temperature. ${ }^{55}$

The iso-stress curves are characterised by an initial step decrease in the modulus (Fig. 4), showing by a step increase in the measured tensile strain, followed by a plateau-like region leading gradually to a sharp decrease in material modulus due to the elastic-plastic transition in the material. The initial step in strain measured corresponds to the liquid crystal to isotropic phase transition within the xLCE, at $\mathrm{T}_{\mathrm{i}}$, and reflects the high stiffness of the smectic LCE compared to the isotropic phase. ${ }^{43,63}$ The temperatures of rapid plastic flow, $\mathrm{T}_{\mathrm{f}}$, were very high in our thiol-epoxy xLCE, all above $200{ }^{\circ} \mathrm{C}$, which promises very little creep at the actuating temperature $\mathrm{T}_{\mathrm{i}}$. The lower $\mathrm{T}_{\mathrm{f}}$ of BD1 over EDDT is rationalised the same way in the $\mathrm{xLCE}$ material as in the isotropic epoxy-thiol elastomers previously studied: a higher content of ester groups within the network resulting in an increased rate of attempts for the BER, a lower modulus of the material, and a lower activation energy of the BER in the network. The highest values of $\mathrm{T}_{\mathrm{f}}$ were obtained for samples containing the short spacer EDT, as its presence in the network reduces the flexibility of polymer chains, compared to longer spacers such as EDDT, leading to stiffer materials; this in turn results in higher flow temperatures as previously demonstrated. ${ }^{55}$

In the context of xLCE, high temperatures of elastic-plastic transition is a desirable property. To enable stable actuation without residual creep, it is necessary to have the temperature range in which bond exchange occurs to be distant from the actuation temperature. This prevents any progressive deterioration of alignment or network topology during the heating required for repeated actuation cycles, whatever the material used. Although $\mathrm{T}_{\mathrm{f}}$ represents the upper limit of temperature for manipulation of the material, and not the onset temperature for the BER, it nevertheless provides an insight into the flow behaviour of the vitrimer.

Programming and thermal actuation. To test the capacity of this epoxy-thiol xLCE family to produce soft actuators, two materials were studied. The EDDT-based material was chosen due to the optimal balance it strikes in transition temperatures: 
an isotropic transition distinctly above room temperature, while maintaining a high stability against plastic flow during actuation. The EDT/EDDT (0.7/0.3) was chosen to compare the effects of the nematic phase (vs. smectic phase of pure EDDT) on the actuation properties.

The samples were aligned post-polymerisation using a creep programming protocol in a DMA instrument, ${ }^{28,36}$ when a constant tensile stress is applied at a high temperature (just below $\mathrm{T}_{\mathrm{f}}$ ), so the plastic creep starts. This process is stopped as the plastic flow reaches the $100 \%$ extension, when we deem a sufficient uniaxial network anisotropy is created. We then cool the sample to ambient temperature and remove the load. The difference between the original and the programmed xLCE, when cooled to room temperature is shown in Fig.5(a). Such an alignment protocol (which does not have to be carried out in DMA:
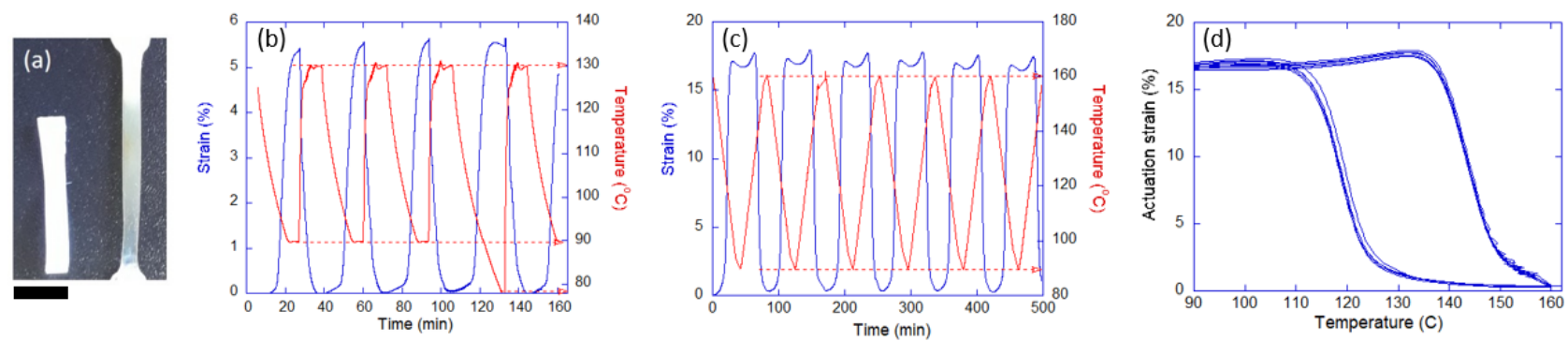

Figure 5. Epoxy-thiol xLCE aligned via plastic creep under tensile load demonstrated stable actuation over multiple cycles. The scale bar $=5 \mathrm{~mm}$. (a) EDDT (smectic) sample before (left) and after (right) the alignment. (b) Thermal cycling of the aligned EDDT sample between $90^{\circ} \mathrm{C}$ and $130{ }^{\circ} \mathrm{C}$ yields a stable smectic-isotropic actuation. (c) Thermal cycling of the aligned EDT/EDDT (0.7/0.3) sample between $90^{\circ} \mathrm{C}$ and $160{ }^{\circ} \mathrm{C}$ yields a stable nematicisotropic actuation. (d) The illustration of 11 thermal cycles on EDT/EDDT (0.7/0.3) sample, from plot (c), showing the overlapping actuation steps.

we often use an oven with a sample under load, visually monitoring its changing length) presents the advantage of reproducibility, when compared to manual alignment, ${ }^{21}$ or alignment that relies on the shape-memory effect in the LC phase ${ }^{42}$ to retain the extended shape. The uniform monodomain xLCE film can be cycled multiple times around the actuation temperature. Overall, a steady actuation was obtained, with the materials yielding a consistent response on thermal each cycle, as illustrated in Fig. 5.

The EDDT sample yielded a relatively low actuation stroke (ca. 5\% strain), Fig. 5(b); this is attributed to the smectic phase of this xLCE material (this amplitude was found to be independent of the temperature at which the alignment was programmed). A wider temperature cycle with deeper cooling ( $4^{\text {th }}$ cycle in the plot) tested the stability of the actuation. The response displayed a plateau in the extended shape, indicating structural stability of the strip of material while it was deep in the smectic phase. In contrast, the nematic sample EDT/EDDT (0.7/0.3) in Fig. 5(c,d) displayed an actuation stroke of approximately 17\%, 3 times higher than for the smectic sample. This was expected, as nematic LCE show higher amplitudes of actuation due to the much less restricted backbone mobility than in the smectic phase. This contrast illustrates the importance of being able to control the phase within a material. 
The full reversibility of actuation is demonstrated by 11 overlapping cycles of heating and cooling in Fig. 5(d). This plot also highlights the notable hysteresis of thermo-mechanical response: on heating, most of the contraction occurs around 140-145 ${ }^{\circ} \mathrm{C}$, while the extension on cooling is around $120^{\circ} \mathrm{C}$. The quasi-equilibrium isotropic transition temperature determined by DSC was around $127^{\circ} \mathrm{C}$, as shown in Table 1 . The reason for such a wide hysteresis is the rate of temperature change, which was $2^{\circ} \mathrm{C} / \mathrm{min}$ in these actuation tests. The macroscopic shape of the LCE network is directly linked to the order parameter (and the response to its change instantaneous), but it takes time for the heat to diffuse through a large sample, to produce the desired change in the order parameter, which is the reason for the observed hysteresis. Very thin films or fibres would have a much narrower hysteresis, as would be the case with much lower heating rate.

\section{CONCLUSIONS}

To conclude, we presented a novel chemistry approach for the synthesis of xLCE with low glass transition, but a high temperature of elastic-plastic transition. The use of an epoxy-thiol "click" chemistry for the polymerisation of LCE vitrimers utilising a transesterification BER yielded materials with an easily controllable network structure. The materials allowed to adjust the isotropic transition $\mathrm{T}_{\mathrm{i}}$ within a wide range of temperatures. The high activation temperature of plastic flow means that an aligned (monodomain) xLCE could be thermally actuated in a stable and repeatable way due to the large gap between the actuation and BER activation temperatures. Different liquid crystalline phases were shown to be accessible by varying the constitution of the network. These results show that a broad range of xLCE materials with diverse properties are accessible through this chemistry approach.

Our aim here was to investigate the options offered by a range of new epoxy-thiol xLCE, not to optimise the actuation strain. No effort was hence directed towards deliberately increasing it. Nevertheless, the spontaneous tensile strain of 10 - $20 \%$ induced on temperature cycling through the nematic-isotropic transition (or the related light-induced actuation, when the sample contains an efficient dye) is a promising platform for many application, in particular, in bending configuration of bi- or multi-layer composites. The dynamic bond-exchange nature of xLCE lends itself to assembling such layered composites due to the covalent bonding forming across surfaces (as well as the exchange in the bulk of the network).

\section{AUTHOR INFORMATION}

\section{Corresponding Author}

*emt1000@cam.ac.uk 
Author Contributions

All authors have contributed to the experimental work, data analysis, and writing of the paper. All authors have given approval to the final version of the manuscript.

Funding Sources

This work was supported by the ERC AdG "APRA" (786659).

Notes

There are no conflicts of interest to declare.

\section{ACKNOWLEDGMENT}

We are grateful to Yan Ji and Yang Yang for useful discussions and advice.

\section{REFERENCES}

(1) Finkelmann, H.; Kock, H.-J.; Rehage, G. Investigations on Liquid Crystalline Polysiloxanes 3. Liquid Crystalline Elastomers - a New Type of Liquid Crystalline Material. Makromol. Chem., Rapid Commun. 1981, 2 (4), $317-322$. https://doi.org/10.1002/marc.1981.030020413.

(2) De Gennes, P. G. Réflexions sur un type de polymeres nematiques. C.R. Acad. Sci. 1975, 281 (B), 101-103.

(3) Küpfer, J.; Finkelmann, H. Nematic Liquid Single Crystal Elastomers. Makromol. Chem., Rapid Commun. 1991, 12 (12), 717-726. https://doi.org/10.1002/marc.1991.030121211.

(4) Hogan, P. M.; Tajbakhsh, A. R.; Terentjev, E. M. Uv Manipulation of Order and Macroscopic Shape in Nematic Elastomers. Phys. Rev. E 2002, 65 (4), 041720. https://doi.org/10.1103/PhysRevE.65.041720.

(5) Winkler, M.; Kaiser, A.; Krause, S.; Finkelmann, H.; Schmidt, A. M. Liquid Crystal Elastomers with Magnetic Actuation. Macromol. Symp. 2010, 291-292 (1), 186-192. https://doi.org/10.1002/masy.201050522.

(6) Huber, J. E.; Fleck, N. A.; Ashby, M. F. The Selection of Mechanical Actuators Based on Performance Indices. Proceedings of the Royal Society of London. Series A: Mathematical, Physical and Engineering Sciences 1997, 453 (1965), $2185-2205$. https://doi.org/10.1098/rspa.1997.0117. 
Robotic Matter with Passive Control of Shape Morphing and Propulsion. Sci. Robot. 2019, 4 (33), eaax7044. https://doi.org/10.1126/scirobotics.aax7044.

(8) Ohm, C.; Brehmer, M.; Zentel, R. Liquid Crystalline Elastomers as Actuators and Sensors. Adv. Mater. 2010, 22 (31), 33663387. https://doi.org/10.1002/adma.200904059.

(9) Babakhanova, G.; Turiv, T.; Guo, Y.; Hendrikx, M.; Wei, Q.-H.; Schenning, A. P. H. J.; Broer, D. J.; Lavrentovich, O. D. Liquid Crystal Elastomer Coatings with Programmed Response of Surface Profile. Nat Commun 2018, 9 (1), 456. https://doi.org/10.1038/s41467-018-02895-9.

(10) Ohzono, T.; Saed, M. O.; Yue, Y.; Norikane, Y.; Terentjev, E. M. Dynamic Manipulation of Friction in Smart Textile Composites of Liquid-Crystal Elastomers. Adv. Mater. Interfaces 2020, 7 (7), 1901996. https://doi.org/10.1002/admi.201901996.

(11) Ferrantini, C.; Pioner, J. M.; Martella, D.; Coppini, R.; Piroddi, N.; Paoli, P.; Calamai, M.; Pavone, F. S.; Wiersma, D. S.; Tesi, C.; Cerbai, E.; Poggesi, C.; Sacconi, L.; Parmeggiani, C. Development of Light-Responsive Liquid Crystalline Elastomers to Assist Cardiac Contraction. Circ Res 2019, 124 (8). https://doi.org/10.1161/CIRCRESAHA.118.313889.

(12) Martella, D.; Parmeggiani, C. Advances in Cell Scaffolds for Tissue Engineering: The Value of Liquid Crystalline Elastomers. Chem. Eur. J. 2018, 24 (47), 12206-12220. https://doi.org/10.1002/chem.201800477.

(13) Warner, M.; Terentjev, E. M. Liquid Crystal Elastomers; International series of monographs on physics; Oxford University Press: Oxford, 2003.

(14) Ware, T. H.; White, T. J. Programmed Liquid Crystal Elastomers with Tunable Actuation Strain. Polym. Chem. 2015, 6 (26), 4835-4844. https://doi.org/10.1039/C5PY00640F.

(15) de Haan, L. T.; Gimenez-Pinto, V.; Konya, A.; Nguyen, T.-S.; Verjans, J. M. N.; Sánchez-Somolinos, C.; Selinger, J. V.; Selinger, R. L. B.; Broer, D. J.; Schenning, A. P. H. J. Accordion-like Actuators of Multiple 3D Patterned Liquid Crystal Polymer Films. Adv. Funct. Mater. 2014, 24 (9), 1251-1258. https://doi.org/10.1002/adfm.201302568.

(16) Yakacki, C. M.; Saed, M.; Nair, D. P.; Gong, T.; Reed, S. M.; Bowman, C. N. Tailorable and Programmable Liquid-Crystalline Elastomers Using a Two-Stage Thiol-Acrylate Reaction. $\quad R S C \quad A d v . \quad 2015, \quad 5 \quad$ (25), 18997-19001. https://doi.org/10.1039/C5RA01039J.

(17) Ware, T. H.; McConney, M. E.; Wie, J. J.; Tondiglia, V. P.; White, T. J. Voxelated Liquid Crystal Elastomers. Science 2015, 347 (6225), 982-984. https://doi.org/10.1126/science.1261019. 
(19) Saed, M. O.; Ambulo, C. P.; Kim, H.; De, R.; Raval, V.; Searles, K.; Siddiqui, D. A.; Cue, J. M. O.; Stefan, M. C.; Shankar, M. R.; Ware, T. H. Molecularly-Engineered, 4D-Printed Liquid Crystal Elastomer Actuators. Adv. Funct. Mater. 2019, 29 (3), 1806412. https://doi.org/10.1002/adfm.201806412.

(20) Davidson, E. C.; Kotikian, A.; Li, S.; Aizenberg, J.; Lewis, J. A. 3D Printable and Reconfigurable Liquid Crystal Elastomers with Light-Induced Shape Memory via Dynamic Bond Exchange. Adv. Mater. 2020, $32 \quad$ (1), 1905682. https://doi.org/10.1002/adma.201905682.

(21) Pei, Z.; Yang, Y.; Chen, Q.; Terentjev, E. M.; Wei, Y.; Ji, Y. Mouldable Liquid-Crystalline Elastomer Actuators with Exchangeable Covalent Bonds. Nature Mater 2014, 13 (1), 36-41. https://doi.org/10.1038/nmat3812.

(22) Montarnal, D.; Capelot, M.; Tournilhac, F.; Leibler, L. Silica-Like Malleable Materials from Permanent Organic Networks. Science 2011, 334 (6058), 965-968. https://doi.org/10.1126/science.1212648.

(23) Fortman, D. J.; Brutman, J. P.; De Hoe, G. X.; Snyder, R. L.; Dichtel, W. R.; Hillmyer, M. A. Approaches to Sustainable and Continually Recyclable Cross-Linked Polymers. ACS Sustainable Chem. Eng. 2018, 6 (9), 11145-11159. https://doi.org/10.1021/acssuschemeng.8b02355.

(24) Pei, Z.; Yang, Y.; Chen, Q.; Wei, Y.; Ji, Y. Regional Shape Control of Strategically Assembled Multishape Memory Vitrimers. Adv. Mater. 2016, 28 (1), 156-160. https://doi.org/10.1002/adma.201503789.

(25) Yang, Y.; Pei, Z.; Li, Z.; Wei, Y.; Ji, Y. Making and Remaking Dynamic 3D Structures by Shining Light on Flat Liquid Crystalline Vitrimer Films without a Mold. J. Am. Chem. Soc. 2016, 138 (7), 2118-2121. https://doi.org/10.1021/jacs.5b12531.

(26) Saed, M. O.; Gablier, A.; Terentejv, E. M. Liquid Crystalline Vitrimers with Full or Partial Boronic-Ester Bond Exchange. Adv. Funct. Mater. 2019, 1906458. https://doi.org/10.1002/adfm.201906458.

(27) Wu, Y.; Yang, Y.; Qian, X.; Chen, Q.; Wei, Y.; Ji, Y. Liquid-Crystalline Soft Actuators with Switchable Thermal Reprogrammability. Angew. Chem. Int. Ed. 2020, 59 (12), 4778-4784. https://doi.org/10.1002/anie.201915694.

(28) Saed, M. O.; Terentjev, E. M. Siloxane Crosslinks with Dynamic Bond Exchange Enable Shape Programming in LiquidCrystalline Elastomers. Sci Rep 2020, 10 (1), 6609. https://doi.org/10.1038/s41598-020-63508-4.

(29) Wang, Z.; Tian, H.; He, Q.; Cai, S. Reprogrammable, Reprocessible, and Self-Healable Liquid Crystal Elastomer with Exchangeable Disulfide Bonds. ACS Appl. Mater. Interfaces 2017, 9 (38), 33119-33128. https://doi.org/10.1021/acsami.7b09246. 
with Versatile Locomotion for Soft Continuum Robots. Adv. Mater. 2018, $30 \quad$ (29), 1801103. https://doi.org/10.1002/adma.201801103.

(31) McBride, M. K.; Martinez, A. M.; Cox, L.; Alim, M.; Childress, K.; Beiswinger, M.; Podgorski, M.; Worrell, B. T.; Killgore, J.; Bowman, C. N. A Readily Programmable, Fully Reversible Shape-Switching Material. Sci. Adv. 2018, 4 (8), eaat4634. https://doi.org/10.1126/sciadv.aat4634.

(32) Ube, T.; Kawasaki, K.; Ikeda, T. Photomobile Liquid-Crystalline Elastomers with Rearrangeable Networks. Adv. Mater. 2016, 28 (37), 8212-8217. https://doi.org/10.1002/adma.201602745.

(33) Chen, Q.; Wei, Y.; Ji, Y. Photo-Responsive Liquid Crystalline Vitrimer Containing Oligoanilines. Chinese Chemical Letters 2017, 28 (11), 2139-2142. https://doi.org/10.1016/j.cclet.2017.09.011.

(34) Chen, Q.; Li, Y.; Yang, Y.; Xu, Y.; Qian, X.; Wei, Y.; Ji, Y. Durable Liquid-Crystalline Vitrimer Actuators. Chem. Sci. 2019, 10 (10), 3025-3030. https://doi.org/10.1039/C8SC05358H.

(35) Kawasaki, K.; Ube, T.; Ikeda, T. Remoldable Crosslinked Liquid-Crystalline Polysiloxane with Side Chain Mesogens Based on Exchangeable Crosslinks. Molecular Crystals and Liquid Crystals 2015, $614 \quad$ (1), $62-66$. https://doi.org/10.1080/15421406.2015.1049910.

(36) Hanzon, D. W.; Traugutt, N. A.; McBride, M. K.; Bowman, C. N.; Yakacki, C. M.; Yu, K. Adaptable Liquid Crystal Elastomers with Transesterification-Based Bond Exchange Reactions. Soft Matter 2018, 14 (6), 951-960. https://doi.org/10.1039/C7SM02110K.

(37) Li, Z.; Yang, Y.; Wang, Z.; Zhang, X.; Chen, Q.; Qian, X.; Liu, N.; Wei, Y.; Ji, Y. Polydopamine Nanoparticles Doped in Liquid Crystal Elastomers for Producing Dynamic 3D Structures. J. Mater. Chem. A 2017, 5 (14), 6740-6746. https://doi.org/10.1039/C7TA00458C.

(38) Pritchard, R. H.; Redmann, A.-L.; Pei, Z.; Ji, Y.; Terentjev, E. M. Vitrification and Plastic Flow in Transient Elastomer Networks. Polymer 2016, 95, 45-51. https://doi.org/10.1016/j.polymer.2016.04.060.

(39) Denissen, W.; Winne, J. M.; Du Prez, F. E. Vitrimers: Permanent Organic Networks with Glass-like Fluidity. Chem. Sci. 2016, 7 (1), 30-38. https://doi.org/10.1039/C5SC02223A. 
Cross-Link Density after Reprocessing: Quantitative Theory and Experiments. Macromolecules 2018 , 51 (15), 5537-5546. https://doi.org/10.1021/acs.macromol.8b00922.

(41) Yu, Z.; Hedden, R. C. Factors Influencing the Shear and Tensile Moduli of Smectic Polydomain Networks. Macromol. Symp. 2013, 329 (1), 125-132. https://doi.org/10.1002/masy.201200101.

(42) Rousseau, I. A.; Mather, P. T. Shape Memory Effect Exhibited by Smectic-C Liquid Crystalline Elastomers. J. Am. Chem. Soc. 2003, 125 (50), 15300-15301. https://doi.org/10.1021/ja039001s.

(43) Osborne, M. J.; Terentjev, E. M. Elasticity of Rubber with Smectic Microstructure. Phys. Rev. E 2000, 62 (4), $5101-5114$. https://doi.org/10.1103/PhysRevE.62.5101.

(44) Lowe, A. B. Thiol-Ene "Click" Reactions and Recent Applications in Polymer and Materials Synthesis. Polym. Chem. 2010, 1 (1), 17-36. https://doi.org/10.1039/B9PY00216B.

(45) Saed, M. O.; Torbati, A. H.; Starr, C. A.; Visvanathan, R.; Clark, N. A.; Yakacki, C. M. Thiol-Acrylate Main-Chain LiquidCrystalline Elastomers with Tunable Thermomechanical Properties and Actuation Strain. J. Polym. Sci. Part B: Polym. Phys. 2017, 55 (2), 157-168. https://doi.org/10.1002/polb.24249.

(46) Martella, D.; Parmeggiani, C.; Wiersma, D. S.; Piñol, M.; Oriol, L. The First Thiol-Yne Click Chemistry Approach for the Preparation of Liquid Crystalline Elastomers. J. Mater. Chem. C 2015, 3 (34), 9003-9010. https://doi.org/10.1039/C5TC01290B.

(47) Yang, H.; Liu, M.-X.; Yao, Y.-W.; Tao, P.-Y.; Lin, B.-P.; Keller, P.; Zhang, X.-Q.; Sun, Y.; Guo, L.-X. Polysiloxane-Based Liquid Crystalline Polymers and Elastomers Prepared by Thiol-Ene Chemistry. Macromolecules 2013, 46 (9), $3406-3416$. https://doi.org/10.1021/ma400462e.

(48) Yang, H.; Wang, L.; Shao, R.; Clark, N. A.; Ortega, J.; Etxebarria, J.; Albouy, P.-A.; Walba, D. M.; Keller, P. Novel LiquidCrystalline Mesogens and Main-Chain Chiral Smectic Thiol-Ene Polymers Based on Trifluoromethylphenyl Moieties. J. Mater. Chem. 2009, 19 (39), 7208. https://doi.org/10.1039/b907907f.

(49) Hoyle, C. E.; Lowe, A. B.; Bowman, C. N. Thiol-Click Chemistry: A Multifaceted Toolbox for Small Molecule and Polymer Synthesis. Chem. Soc. Rev. 2010, 39 (4), 1355. https://doi.org/10.1039/b901979k.

(50) Fernández-Francos, X.; Konuray, A.-O.; Belmonte, A.; De la Flor, S.; Serra, À.; Ramis, X. Sequential Curing of Off-Stoichiometric Thiol-Epoxy Thermosets with a Custom-Tailored Structure. Polym. Chem. 2016, 7 (12), 2280-2290. https://doi.org/10.1039/C6PY00099A. 
ties of Novel Polyesters Based on Dithiotriethylene Glycol. European Polymer Journal 2006, 42 (12), $3374-3382$. https://doi.org/10.1016/j.eurpolymj.2006.08.022.

(52) Brändle, A.; Khan, A. Thiol-Epoxy 'Click’ Polymerization: Efficient Construction of Reactive and Functional Polymers. Polym. Chem. 2012, 3 (12), 3224. https://doi.org/10.1039/c2py20591b.

(53) Konuray, A. O.; Fernández-Francos, X.; Ramis, X. Latent Curing of Epoxy-Thiol Thermosets. Polymer 2017, 116, 191203. https://doi.org/10.1016/j.polymer.2017.03.064.

(54) Belmonte, A.; Russo, C.; Ambrogi, V.; Fernández-Francos, X.; De la Flor, S. Epoxy-Based Shape-Memory Actuators Obtained via Dual-Curing of Off-Stoichiometric “Thiol-Epoxy” $\quad$ Mixtures. $\quad$ Polymers $\quad 2017,9 \quad$ (12), 113. https://doi.org/10.3390/polym9030113.

(55) Gablier, A.; Saed, M. O.; Terentjev, E. Rates of Transesterification in Epoxy-Thiol Vitrimers. Soft Matter 2020, 16 (22), 5195-5202. https://doi.org/10.1039/D0SM00742K.

(56) Hayashi, M.; Katayama, A. Preparation of Colorless, Highly Transparent, Epoxy-Based Vitrimers by the Thiol-Epoxy Click Reaction and Evaluation of Their Shape-Memory Properties. ACS Appl. Polym. Mater. 2020, $2,2452-2457$. https://doi.org/10.1021/acsapm.0c00397.

(57) Matejka, L.; Pokomy, S.; Dusek, K. Network Formation Involving Epoxide and Carboxyl Groups. Polym. Bull. 1982, 7 , 123-128. https://doi.org/10.1007/BF00265462.

(58) Demongeot, A.; Mougnier, S. J.; Okada, S.; Soulié-Ziakovic, C.; Tournilhac, F. Coordination and Catalysis of Zn ${ }^{2+}$ in Epoxy-Based Vitrimers. Polym. Chem. 2016, 7 (27), 4486-4493. https://doi.org/10.1039/C6PY00752J.

(59) Li, Y.; Badrinarayanan, P.; Kessler, M. R. Liquid Crystalline Epoxy Resin Based on Biphenyl Mesogen: Thermal Characterization. Polymer 2013, 54 (12), 3017-3025. https://doi.org/10.1016/j.polymer.2013.03.043.

(60) Giamberjni, M.; Amendola, E.; Carfagna, C. Liquid Crystalline Epoxy Thermosets. Molecular Crystals and Liquid Crystals Science and Technology. Section A. Molecular Crystals and Liquid Crystals 1995, $266 \quad$ (1), 9-22. https://doi.org/10.1080/10587259508033628.

(61) Gonzalez, M. G.; Cabanelas, J. C.; Baselga, J. Chapter 13: Applications of FTIR on Epoxy Resins - Identification, Monitoring and Curing Process, Phase Separation and Water Uptake. In Infrared Spectroscopy: Materials Science, Engineering and Technology; 2012. 
(62) Giamberini, M.; Amendola, E.; Carfagna, C. Lightly Crosslinked Liquid Crystalline Epoxy Resins: The Effect of RigidRod Length and Applied Stress on the State of Order of the Cured Thermoset. Macromol. Chem. Phys. 1997, 198 (10), 3185-3196. https://doi.org/10.1002/macp.1997.021981016.

(63) Nishikawa, E.; Finkelmann, H. Orientation Behavior of Smectic Polymer Networks by Uniaxial Mechanical Fields. Macromol. Chem. Phys. 1997, 198 (8), 2531-2549. https://doi.org/10.1002/macp.1997.021980814. 